\title{
Bounds for the Second Largest Eigenvalue of Real $3 \times 3$ Symmetric Matrices with Entries Symmetric about the Origin
}

\author{
Barini Geoffrey $^{1}$, Kivunge Benard ${ }^{2}$, Jotham Akanga ${ }^{3}$ \\ ${ }^{1,3}$ Department of Pure and Applied Mathematics, Jomo Kenyatta University of Agriculture and Technology, Nairobi, Kenya \\ ${ }^{2}$ Department of Pure and Applied Mathematics, Kenya Polytechnic University College, Nairobi, Kenya \\ Email: geoffreyonkundi@yahoo.com
}

Received April 3, 2012; revised May 3, 2012; accepted May 10, 2012

\section{ABSTRACT}

Let $A_{S_{n}[a, b]}$ denote a set of all real nxn symmetric matrices with entries in the interval $[a, b]$. In this article, we present bounds for the second largest eigenvalue $\lambda_{2}(A)$ of a real symmetric matrix $\# \mathbf{1}$, such that $\boldsymbol{A} \in A_{S_{3}[-b, b]}$.

Keywords: Bounds; Determinant; Eigenvalues; Trace

\section{Introduction}

Throughout this article, $A_{S_{n}[a, b]}$ denotes a set of $\boldsymbol{n} \times \boldsymbol{n}$ real symmetric matrices whose entries are in the interval $[\boldsymbol{a}, \boldsymbol{b}]$. Eigenvalues of any real $\boldsymbol{n} \times \boldsymbol{n}$ symmetric matrix $A$, will be represented by

$$
\lambda_{1}(A) \geq \lambda_{2}(A) \geq \cdots \geq \lambda_{n}(A) .
$$

The smallest $\lambda_{n}(\boldsymbol{A})$ and the largest $\lambda_{1}(\boldsymbol{A})$ eigenvalues have been studied extensively in the recent decades. Recently, many researchers have turned attention to the second largest eigenvalue $\lambda_{2}(A)$ due to its applications in science and engineering. For example, thesecond largest eigenvalue governs the rate at which the statistics of the Markov chain converge to equilibrium. Here, we investigate bounds for $(\boldsymbol{A})$ when entries of $\boldsymbol{A}$ vary in the interval $[-\boldsymbol{b}, \boldsymbol{b}]$. then

In 1985, Constantine [1] showed that if $\boldsymbol{A} \in \boldsymbol{A}_{S_{n}[0, b]}$,

$$
\lambda_{n}(A) \geq \frac{-n b}{2} \text { and } \lambda_{n}(A) \geq \frac{-\sqrt{\left(n^{2}-1\right) b^{2}}}{2}
$$

if $n$ is even and odd respectively. Similar results are presented in [2]. In [3], Zhan gave bounds for both the largest eigenvalue $\lambda_{1}(A)$ and the smallest eigenvalue $\lambda_{n}(A)$ when entries of $A$ are in a general interval $[a, b]$. In the same paper [3], Zhan posed the following problem: For a given integer $j$ with $2 \leq j \leq n-1$, find

$$
\begin{aligned}
& \max \left\{\lambda_{j}(\boldsymbol{A}): \boldsymbol{A} \in A_{S_{n}[a, b]}\right\}, \\
& \min \left\{\lambda_{j}(\boldsymbol{A}): \boldsymbol{A} \in A_{S_{n}[a, b]}\right\} .
\end{aligned}
$$

We are concerned with the case $j=2$ when $\boldsymbol{A} \in A_{S_{3}[-b, b]}$. We employ analytical approach discussed in [4] and the properties

$$
\begin{aligned}
& \operatorname{tr} A=\lambda_{1}(A)+\lambda_{2}(A)+\cdots+\lambda_{n}(A), \\
& \operatorname{det} A=\lambda_{1}(A) \lambda_{2}(A) \cdots \lambda_{n}(A)
\end{aligned}
$$

to determine these bounds. The following result will prove useful later. If $A=\left(a_{i j}\right)$ is any real $3 \times 3$ matrix such that $\left|a_{i j}\right| \leq 1$, then $|\operatorname{det} A| \leq 4 \quad[5,6]$. It immediately follows that if $\boldsymbol{A} \in A_{S_{3}[-b, b]}$, then

$$
|\operatorname{det} A| \leq 4 b^{3}
$$

This paper is organized as follows. In Section 2, analytical method for eigenvalues of real $3 \times 3$ symmetric matrices is discussed. In Section 3, we derive bounds for $\lambda_{2}(A)$. Finally, a numerical example is given in Section 4.

\section{Analytical Calculation of Eigenvalues}

A detailed description of this technique can be found in [4]. Let

$$
\boldsymbol{A}=\left[\begin{array}{lll}
a_{11} & a_{12} & a_{13} \\
a_{12} & a_{22} & a_{23} \\
a_{13} & a_{23} & a_{33}
\end{array}\right]
$$

be a real $3 \times 3$ symmetric matrix. Eigenvalues of $A$ can be directly calculated by solving the corresponding characteristic equation

$$
k(\lambda)=\lambda^{3}+c_{2} \lambda^{2}+c_{1} \lambda+c_{0}=0
$$


where,

$$
\begin{aligned}
& c_{2}=-a_{11}-a_{22}-a_{33} \\
& c_{1}=a_{11} a_{22}+a_{11} a_{33}+a_{22} a_{33}-a_{12}^{2}-a_{13}^{2}-a_{23}^{2} \\
& c_{0}=a_{11} a_{23}^{2}+a_{22} a_{13}^{2}+a_{33} a_{12}^{2}-a_{11} a_{22} a_{33}-2 a_{12} a_{13} a_{23}
\end{aligned}
$$

Equation (2.2) is then solved by first depressing it, i.e., transforming it to the form

$$
x^{3}-3 x=2 p^{-\frac{3}{2}} q
$$

with,

$$
\begin{aligned}
& p=c_{2}^{2}-3 c_{1}, \\
& q=-\frac{27}{2} c_{0}-c_{2}^{3}+\frac{9}{2} c_{2} c_{1}, \\
& x=\frac{3}{\sqrt{p}}\left(\lambda+\frac{1}{3} c_{2}\right) .
\end{aligned}
$$

Solutions to Equation (2.4) are given by

$$
\begin{aligned}
& x_{1}=2 \cos \theta, \\
& x_{2}=-\cos \theta+\sqrt{3} \sin \theta, \\
& x_{3}=-\cos \theta-\sqrt{3} \sin \theta .
\end{aligned}
$$

where,

$$
\theta=\frac{1}{3} \tan ^{-1} \frac{\sqrt{p^{3}-q^{2}}}{q}
$$

Finally, eigenvalues of $A$ becomes

$$
\lambda_{i}(A)=x_{i} \frac{\sqrt{p}}{3}-\frac{1}{3} c_{2} \text {, for } i=1,2,3 .
$$

\section{Bounds for the Second Largest Eigenvalue $\lambda_{2}(A)$}

Note that $x_{2}=-\cos \theta+\sqrt{3} \sin \theta$, corresponds to the second largest eigenvalue. We therefore determine the values of $x_{2}, p$ and $c_{2}$ which minimizes or maximizes $\lambda_{2}(A)$. However, this is not straight forward since $x_{2}, p$ and $c_{2}$ depends on the entries of $A$ which vary in the interval $[-b, b]$. We shall heavily rely on minimizing or maximizing $-c_{2}$.

For the lower bound we require the largest possible value of $p$ such that $x_{2}$ and $-c_{2}$ are minimum. Observe that if we put $a_{i i}=-b$, then

$$
\begin{aligned}
& \min \left(-c_{2}\right)=\min (\operatorname{tr} A)=-3 b, \\
& p=3\left(a_{12}^{2}+a_{13}^{2}+a_{23}^{2}\right), \\
& q=27 a_{12} a_{13} a_{23} .
\end{aligned}
$$

Setting $\left|a_{i j}\right|=\left|a_{k j}\right|=\left|a_{i k}\right|=b$ such that

$$
a_{i j} a_{k j} a_{i k}=b^{3}
$$

where $1 \leq i, j, k \leq 3$ with $i \neq j \neq k$ we obtain,

$$
p=9 p^{2} \text { and } q=27 b^{3} .
$$

Thus $x_{2}=x_{3}=-1$, as required. These correspond to the eigenvalues:

$$
\lambda_{2}(A)=\lambda_{3}(A)=-2 b
$$

Now, suppose there exist $\lambda_{2}^{*}(A)$ and $\lambda_{3}^{*}(A)$ such that

$$
\lambda_{2}(A)>\lambda_{2}^{*}(A)=(-2 b-\delta) \geq \lambda_{3}^{*}(A)=(-2 b-\epsilon),
$$

for some real numbers $\epsilon \geq \delta>0$. Note that

$$
\operatorname{tr} A=\lambda_{1}^{*}(A)+\lambda_{2}^{*}(A)+\lambda_{3}^{*}(A) \geq-3 b
$$

Therefore we must have $\lambda_{1}^{*}(A)>b$. However, this is impossible since from (1.5) we have

$$
\lambda_{1}^{*}(A)=\frac{\operatorname{det} A}{\lambda_{2}^{*}(A) \lambda_{3}^{*}(A)}=\frac{\operatorname{det} A}{4 b^{2}+2 b(\epsilon+\delta)+\epsilon \delta}<b .
$$

We thus deduce that $\lambda_{2}(A) \geq-2 b$. Equality is attained by the following matrices:

$$
\begin{aligned}
& {\left[\begin{array}{ccc}
-b & b & b \\
b & -b & b \\
b & b & -b
\end{array}\right],\left[\begin{array}{ccc}
-b & -b & -b \\
-b & -b & b \\
-b & b & -b
\end{array}\right],} \\
& {\left[\begin{array}{ccc}
-b & -b & b \\
-b & -b & -b \\
b & -b & -b
\end{array}\right],\left[\begin{array}{ccc}
-b & b & -b \\
b & -b & -b \\
-b & -b & -b
\end{array}\right] .}
\end{aligned}
$$

Similarly, for the upper bound, we require the largest possible value of $p$ such that $x_{2}$ and $-c_{2}$ are maximum. Note that setting $a_{i i}=b$ yields

$$
\begin{aligned}
& \max \left(-c_{2}\right)=\max (\operatorname{tr} A)=3 b, \\
& p=3\left(a_{12}^{2}+a_{13}^{2}+a_{23}^{2}\right), \\
& q=27 a_{12} a_{13} a_{23} .
\end{aligned}
$$

Now, if we put $\left|a_{i j}\right|=\left|a_{k j}\right|=\left|a_{i k}\right|=b$ such that

$$
a_{i j} a_{k j} a_{i k}=-b^{3}
$$

where $1 \leq i, j, k \leq 3$ with $i \neq j \neq k$ we have,

$$
p=9 b^{2} \text { and } q=-27 b^{3} .
$$

Check that

$$
\theta=\frac{1}{3} \tan ^{-1} \frac{\sqrt{\left(9 b^{2}\right)^{3}-\left(-27 b^{3}\right)^{2}}}{-27 b^{3}}=60^{\circ}
$$

and hence $x_{1}=x_{2}=1$, corresponding to the eigenvalues:

$$
\lambda_{1}(A)=\lambda_{2}(A)=2 b \text {. }
$$

Again, assume there exist $\lambda_{1}^{*}(A)$ and $\lambda_{2}^{*}(A)$ such that

$$
\lambda_{2}(A)<\lambda_{2}^{*}(A)=(2 b+\delta) \leq \lambda_{1}^{*}(A)=(2 b+\epsilon),
$$


for some real numbers $\epsilon \geq \delta>0$. Considering the fact

$$
\operatorname{tr} A=\lambda_{1}^{*}(A)+\lambda_{2}^{*}(A)+\lambda_{3}^{*}(A) \leq 3 b,
$$

we necessarily have $\lambda_{3}^{*}(A)<-b$. Again from (1.5) we obtain

$$
\lambda_{3}^{*}(A)=\frac{\operatorname{det} A}{\lambda_{1}^{*}(A) \lambda_{2}^{*}(A)}=\frac{\operatorname{det} A}{4 b^{2}+2 b(\epsilon+\delta)+\epsilon \delta}>-b .
$$

This is a contradiction and hence we conclude that $\lambda_{2}(A) \leq 2 b$. Equality is attained by the following matrices:

$$
\begin{aligned}
& {\left[\begin{array}{ccc}
b & -b & -b \\
-b & b & -b \\
-b & -b & b
\end{array}\right],\left[\begin{array}{ccc}
b & b & -b \\
b & b & b \\
-b & b & b
\end{array}\right],} \\
& {\left[\begin{array}{ccc}
b & b & b \\
b & b & -b \\
b & -b & b
\end{array}\right],\left[\begin{array}{ccc}
b & -b & b \\
-b & b & b \\
b & b & b
\end{array}\right] .}
\end{aligned}
$$

\section{Numerical Example}

Let $\boldsymbol{A} \in A_{S_{3}[-2.3,2.3]}$. We first consider the lower bound for $\lambda_{2}(A)$. According to Equation (2.8), we require $a_{11}=a_{22}=a_{33}=-2.3$, so that

$$
\begin{aligned}
& \min \left(-c_{2}\right)=\min (\operatorname{tr} A)=-6.9, \\
& c_{1}=15.87-a_{12}^{2}-a_{13}^{2}-a_{23}^{2} \\
& c_{0}=-2.3\left(a_{23}^{2}+a_{13}^{2}+a_{12}^{2}\right)+12.167-2 a_{12} a_{13} a_{23} .
\end{aligned}
$$

Substituting (4.1) into (2.5) yields

$$
\begin{aligned}
& p=3\left(a_{12}^{2}+a_{13}^{2}+a_{23}^{2}\right), \\
& q=27 a_{12} a_{13} a_{23} .
\end{aligned}
$$

Now, $p$ is maximum when $a_{12}=a_{13}=a_{23}= \pm 2.3$. However, by noting that $-1 \leq x_{2} \leq 1$, we require $q>0$. Thus we must have $a_{12} a_{13} a_{23}=2.3^{3}=12.167$, with $a_{i j}= \pm 2.3$. Finally, from (2.6), (2.7) and (4.2), we easily have $x_{2}=x_{3}=-1$, corresponding to the eigenvalues $\lambda_{2}(A)=\lambda_{3}(A)=-4.6$. We now let $\lambda_{2}^{*}(A)$ and $\lambda_{3}^{*}(A)$ be eigenvalues such that

$$
\lambda_{2}(A)>\lambda_{2}^{*}(A)=(-4.6-\delta) \geq \lambda_{3}^{*}(A)=(-4.6-\epsilon),
$$

for some real numbers $\epsilon \geq \delta>0$. It immediately implies that

$$
\lambda_{1}^{*}(A)+\lambda_{2}^{*}(A)+\lambda_{3}^{*}(A) \geq-6.9
$$

However, (4.3) is valid only if $\lambda_{1}^{*}(A)>2.3$. Applying (1.4) results in

$$
\lambda_{1}^{*}(A)=\frac{\operatorname{det} A}{\lambda_{2}^{*}(A) \lambda_{3}^{*}(A)}=\frac{48.668}{21.16+4.6(\epsilon+\delta)+\epsilon \delta}<2.3
$$

Note that

$$
\operatorname{det} A=-C_{0}=48.668=2.3^{3}\left|\operatorname{det} A^{*}\right|=2.3^{3}(4),
$$

where $\left|\operatorname{det} A^{*}\right|$ is the maximum determinant of a real 3 $\times 3$ matrix whose entries are in a unit closed disc. Thus $\lambda_{2}(A) \geq-4.6$. The minimizing matrices readily follow from (3.8). For the upper bound we set $a_{11}=a_{22}=a_{33}=$ 2.3, giving

$$
\begin{aligned}
& \max \left(-c_{2}\right)=\max (\operatorname{tr} A)=6.9, \\
& c_{1}=15.87-a_{12}^{2}-a_{13}^{2}-a_{23}^{2}, \\
& c_{0}=2.3\left(a_{23}^{2}+a_{13}^{2}+a_{12}^{2}\right)-12.167-2 a_{12} a_{13} a_{23} .
\end{aligned}
$$

Substituting (4.4) into (2.5) results in

$$
\begin{aligned}
& p=3\left(a_{12}^{2}+a_{13}^{2}+a_{23}^{2}\right), \\
& q=27 a_{12} a_{13} a_{23} .
\end{aligned}
$$

It is easy to check that if $a_{12}=a_{13}=a_{23}= \pm 2.3$, such that $a_{12} a_{13} a_{23}=-12.167$, then $x_{1}=x_{2}=1$. This correspond to the eigenvalues $\lambda_{1}(A)=\lambda_{2}(A)=4.6$. Similarly if we let $\lambda_{1}^{*}(A)$ and $\lambda_{2}^{*}(A)$ such that

$$
\lambda_{2}(A)<\lambda_{2}^{*}(A)=(4.6+\delta) \leq \lambda_{1}^{*}(A)=(4.6+\epsilon),
$$

then

$$
\lambda_{1}^{*}(A)+\lambda_{2}^{*}(A)+\lambda_{3}^{*}(A) \leq 6.9
$$
ever,

Check that (4.6) holds only if $\lambda_{3}^{*}(A)<-2.3$. How-

$$
\lambda_{3}^{*}(A)=\frac{\operatorname{det} A}{\lambda_{1}^{*}(A) \lambda_{2}^{*}(A)}=\frac{-48.668}{21.16+4.6(\epsilon+\delta)+\epsilon \delta}>-2.3
$$

Thus $\lambda_{2}(A) \leq 4.6$ and the maximizing matrices follow from (3.17).

\section{Acknowledgements}

Thanks are due to the late Professor Cecilia Mwathi for her support during the initial stages of this research.

\section{REFERENCES}

[1] G. Constantine, "Lower Bounds for the Spectra of Symmetric Matrices with Nonnegative Entries," Linear Algebra and its Applications, Vol. 65, 1985, pp. 171-178. doi:10.1016/0024-3795(85)90095-3

[2] R. Roth, “On the Eigenvectors Belonging to the Minimum Eigenvalue of an Essentially Nonnegative Symmetric Matrix with Bipartite Graph,” Linear Algebra and Its Applications, Vol. 118, 1989, pp. 1-10. doi:10.1016/0024-3795(89)90569-7.

[3] X. Zhan, "Extremal Eigenvalues of Real Symmetric Matrices with Entries in an Interval," Siam Journal of Matrix Analysis and Applications, Vol. 27, No. 3, 2006, pp. 851-860. doi:10.1137/050627812 
[4] J. Kopp, "Efficient Numerical Diagonalization of $3 \times 3$ Hermitian Matrices," International Journal of Modern Physics C, Vol. 19, No. 3, 2008, pp. 523-548. doi:10.1142/S0129183108012303

[5] J. Brenner, "Hadamard Maximum Determinant Problem,"
The American Mathematical Monthly, Vol. 79, No. 6, 1972, pp. 626-630.

[6] N. J. A. Sloan and P. Simon, "The Encyclopaedia of Integer Sequences,” Academic Press Inc., London, 1995. 